\title{
A Fast, Multi-class Algorithm for Classifying Aerial Images
}

(航空写真のための高速画像分類)

John W. Gates ${ }^{\dagger}$, Miki Haseyama (member) ${ }^{\dagger}$, Hideo Kitajima (member) ${ }^{\dagger}$

\begin{abstract}
An approach is described for classifying images that does not require first dividing an image into small blocks and then classifying it based on the features of the individual blocks, as do traditional approaches. Instead the line features of the entire image are extracted and used, along with the pixel intensity, to classify each pixel in the image. To increase the classification speed, a fast line-extraction algorithm has been developed that extracts the line features directly from the original image without pre-processing. A classification tree with single variable splits is used to classify the image. Testing of a five-class aerial-image classification algorithm showed that it had an average error rate of $17.6 \%$. Running on a $600-\mathrm{MHz}$ Pentium III processor, it had an average classification time of 2.18 seconds for $512 \times 512$ grayscale images. This approach can be used for many different applications by training the classification tree with the desired classes.
\end{abstract}

Key words: image classification, line extraction, aerial images, classification trees, and multi-class classification

\section{Introduction}

The classification of images is one of the most important areas in the field of image processing. The applications of image classification stretch from the extremes of land-use classification from satellite images ${ }^{1)}$ to classifying cells in medical images ${ }^{2}$. Other applications are fingerprint analysis ${ }^{3)}$, face recognition ${ }^{4)}$, and scanning image databases ${ }^{5}$. Although this field has many promising applications, there are fundamental problems that make it very difficult. The major difficulty is that images are very complex and the information they contain is distributed in at least two dimensions. Also, to classify the objects, care must be taken to observe the information in a large area around the object.

All image classification systems can be divided into two sequential stages ${ }^{6}$. The first stage is to extract the features necessary for the classification from the input image, and the second is to classify the image based on the extracted features. Since the accuracy of the first stage will directly impact on the second stage, care must be taken in the selection of the classification feature set.

One of the traditional approaches to extracting the classification features from the image is to subdivide

Received Aug. 22, 2001; Revised Dec. 12, 2001; Accepted Jan. 10, 2002

$\dagger$ Hokkaido University

(N-13 W-8 Kita-ku, Sapporo, 080-8628, Japan) the image into smaller blocks and then use the statistical features of the pixels within the block ${ }^{7) 8}$. However, it has been argued that these small windows are inadequate for image classification ${ }^{9)}$ as they examine the local properties of the block and exclude information outside the block. Another factor that must be considered is the type of features that will be used for classification. Some of the most common types include, DC$\mathrm{T}$ coefficients $^{10) 11}$, color histograms ${ }^{5) 11}$, edge direction histograms ${ }^{11)}$, shape $^{5)}$, intensity ${ }^{5)}$, texture ${ }^{5)}$ and linefeatures ${ }^{12)}$. All of these features can be used for classifying images, however for high-speed and high-accuracy applications certain features are more useful than others. Although color information is very useful, the number of color images is much smaller than the number of grayscale images, thus relying on color severely limits the range of applications. Other features, such as histograms, texture and DCT coefficients have a high computation cost when the window size is large.

Many different algorithms have been proposed for classifying images. Some of the most common algorithms are; neural networks ${ }^{113) 14}$, nearest-neighbor classifiers $^{15)}$, hidden Markov models ${ }^{10) 16) 17)}$, decision trees $^{6) 18)}$ and even multiple classifiers ${ }^{1920)}$. All of these methods have their advantages and disadvantages, so the choice of classifier will be determined by the application. The performance of all the classifiers is depen- 
dent on the extraction of reasonable features and the accuracy of the training.

High-speed aerial image classification was chosen as a reasonable application to demonstrate the proposed classification algorithm. This is a very important application for monitoring changes in the environment. Recently, the Kyoto Accord on Global Warming was signed by many nations. In order to enforce the regulations of the Accord, it is necessary to monitor changes in the size of forests. This will require millions of satellite images to be classified, thus a high-speed classification algorithm is required. There are also other organizations that require vast amounts of aerial images to be processed. For example, the Geographical Survey Institute of Japan ${ }^{21)}$ plans to publish 950,000 sheets of aerial images. In the proposed algorithm the regions of the aerial image are classified into five classes. These classes are fields/beaches, buildings/roads, water, forests and shadows.

To avoid the problems associated with block-based image classification algorithms, we chose to use globally extracted line-features and pixel intensity as a reasonable feature set for the classifier. The line-features used in the classification feature set are the length of the line, the slope of the line in relation to the neighboring lines, and the distance from the pixel to the line for the five lines closest to the pixel. The classifier chosen was a classification tree using single variable splits ${ }^{22) 23}$. This type of classifier was chosen because it is simple to train and has a high classification speed.

Since line-features were chosen as part of the classification feature set, the choice of the line extraction algorithm becomes an issue. There are several popular line extraction methods, such as the Hough transform ${ }^{24)-26)}$ and its derivatives ${ }^{27)-30)}$, chain $\operatorname{code}^{31) 32)}$, polygon approximation $^{33) 34)}$ and other methods ${ }^{2425) 35)}$. Since all of these algorithms use pre-processing to extract the edge pixels from the image, the total line extraction time becomes excessive. Therefore we have designed a simple, high-speed line extraction algorithm that extracts the line-features directly from the original image.

In the following section the three major components of the classification algorithm will be described in detail. Section 2.2 describes a fast line extraction algorithm that does not require pre-processing. In Section 2.3 the line-feature distribution algorithm is described. This algorithm is used instead of windowing for distributing the line-features to the neighboring pixels. In Section 2.4 the classification tree and its training are described.
In Section 3 experimental results are shown to demonstrate the strengths and weaknesses of the algorithm. Finally the conclusions are presented in Section 4.

\section{The Classification Algorithm}

\subsection{Overview}

All classification algorithms can be divided into two stages; the extraction of the classification feature set and the classification based on the extracted features. Further stages may refine the classification or extract a different set of features or even re-train the classifier. However, the first two stages, extraction and classification, must be present in all systems and together form the simplest classifier.

The problem with block-based classification algorithms is that the artificial partitioning usually cuts important features or omits them entirely resulting in errors in the feature set and subsequent errors in the classifier. In order to avoid this partitioning problem, the proposed algorithm uses only globally extracted features to form the feature set. By experimentation, it was found that line-features and pixel intensity form a sufficient set of features for accurate classification by the second stage. To increase the speed of the first stage, two component algorithms, which operate simultaneously, were used to form the feature set. The first component algorithm is the fast line extraction algorith$m$ described below. When this algorithm has extracted a line, the second component algorithm distributes the line-feature information to the neighboring pixels and then the line extraction algorithm will extract another line and the process will repeat until all the lines have been extracted from the image. This line-feature distribution algorithm is described in detail in Section 2.3.

Only one algorithm is needed in the classification stage. A classification tree with single variable splits was chosen based on the nature of the application. A classification tree is a robust classifier in the presence of errors in the training set. This is important, as it is difficult to classify aerial images by hand. Also, once the tree has been computed, it can quickly classify the complex feature set. The classification tree and the training of the tree are described in detail in Section 2.4.

\subsection{Line Extraction}

Any line extraction algorithm that extracts the linefeatures necessary for the classification of the image may be used. However, to achieve high-speed image classification, this is one component of the classification algorithm where a significant amount of time can be saved. 
The traditional line extraction algorithms use a twostep approach to extract lines. The first step is to extract the edge pixels and the second step is to form lines with the extracted edge pixels. This pre-processing first step is used to reduce the amount of information that needs to be processed and to compensate for blurred edge transitions that are common in natural images. The problem with this pre-processing is that it requires a significant amount of processing time to extract the desired edges and then to remove the extraneous edges that may have been extracted. A more serious problem with the pre-processing is that it removes the edge pixel$s$ from the intensity information and therefore complex algorithms, which need a large amount of processing time, are required to connect the extracted edge pixels into lines.

To demonstrate these problems, the images shown in Figs. 4 to 6 were tested using the Standard Hough Transform ${ }^{24) 36) 37)}$ (SHT) and the Windowing Hough Transfor$\mathrm{m}^{30) 38)}$ (WHT), which are two of the conventional line extraction algorithms. For the pre-processing, the Sobel operator $^{39)}$ was used to extract the edge pixels and an erosion filter ${ }^{39)}$ was used to reduce the extraneous edge pixels. It is necessary to remove the extraneous pixels as the line extraction time is directly proportional to the number of pixels and the extraneous pixels also reduce the accuracy of the extracted lines. The results of the conventional algorithms are recorded in Table 1. By comparing Table 1 with the results of the proposed line extraction algorithm given in Table 3 , it can be seen that the average line extraction time is approximately the same as the pre-processing time. Also the total line extraction time is 10 times faster than the WHT. The reason that the line extraction time is faster than the pre-processing time for Fig. 4 is that the Sobel operator tests the eight neighboring pixels for every pixel. Whereas the proposed algorithm only tests two pixels in the large uniform intensity regions in the image, thus reducing the computation cost.

In the proposed classification algorithm a different approach to line extraction has been used. The new approach is to extract the line segments directly from the original image without pre-processing. This approach leads to a significant improvement in the speed of the line extraction and the extracted line-features are sufficient for classifying the image. The new approach is based on the assumption that lines occur at the border between two regions of different intensity within the image. This basic assumption leads directly to a
Table 1 Conventional line extraction results.

\begin{tabular}{c|c|c|c|c} 
Table 1 Conventional line extraction results. \\
\hline \hline & Fig. 4 & Fig. 5 & Fig. 6 & Ave. \\
\hline Sobel only (sec) & 0.0822 & 0.0857 & 0.0854 & 0.0844 \\
\hline Sobel only (pixel) & 41671 & 103854 & 94051 & 79859 \\
\hline Sobel + Erosion (sec) & 0.0997 & 0.1049 & 0.1045 & 0.103 \\
\hline Sobel + Erosion (pixel) & 18668 & 40407 & 28426 & 29167 \\
\hline SHT time (sec) & 743.3 & 1203.7 & 1099.8 & 1015.6 \\
\hline SHT lines & 2501 & 5794 & 3501 & 3932 \\
\hline SHT quality & good & good & good & good \\
\hline SHT + pre-processing (sec) & 743.4 & 1203.8 & 1099.9 & 1015.7 \\
\hline WHT time (sec) & 0.5309 & 1.337 & 0.7467 & 0.8715 \\
\hline WHT lines & 4397 & 9418 & 5780 & 6531 \\
\hline WHT quality & poor & poor & poor & poor \\
\hline WHT + pre-processing (sec) & 0.6306 & 1.442 & 0.8512 & 0.9746 \\
\hline
\end{tabular}

line extraction algorithm with the following steps:

(1) Locate a region of uniform intensity.

(2) Trace the perimeter of the region and record the coordinates of the pixels.

(3) Form lines from this set of correlated pixel coordinates.

(4) Repeat steps 1 through 3 for the next region of uniform intensity.

To locate a region of uniform intensity the line extraction algorithm scans pixel by pixel across the image looking for a significant contrast in the intensity, either horizontally or vertically, between the present pixel and its neighbor. If a significant contrast exists then the border of a region of uniform intensity has been found. In the proposed line extraction algorithm the minimum difference in the intensity between pixels must be at least thirty. However, this is a parameter that can be changed for various applications and the value used was chosen based on experimentation. If the value of this threshold is too low, then many extraneous lines will be extracted and if the value is too high, then some important lines will be lost. This value remained constant for all the training images and test images. In aerial images, one pixel represents a significant distance. Therefore intensity transitions occur abruptly thus justifying this simple comparison between adjacent pixels. However, in other applications where intensity transitions occur over a number of pixels, it is necessary to use an approach that integrates the change in intensity over a limited region to determine if an intensity transition exists.

Once a new region of uniform intensity has been located it is necessary to trace the perimeter of the region and record the coordinates of the pixels forming the perimeter. Since the region is defined as an area of relatively uniform intensity, if the perimeter is traced in a clockwise direction, then the nearest counter clock- 
wise pixel to the direction of movement must be outside the intensity range of the region. This rule will allow the line extraction algorithm to trace the region and arrive back at the starting point. As the line extraction algorithm traces the perimeter of the region, it records the coordinates of the pixels in a list. This list is highly correlated as each pixel is sequential and belongs to the same region of uniform intensity. Also, each pixel is marked as a processed pixel to prevent duplication in the line extraction.

Once the pixels of the perimeter of the region have been extracted, it is necessary to form them into lines. Due to the high correlation between pixels this is a simple process. In the proposed line extraction algorith$\mathrm{m}$ simple linear regression ${ }^{40)}$ is used. Linear regression allows the mean-square error (MSE) to be calculated easily so this becomes a reasonable estimate for starting and ending the line segments. Therefore, to form the line segments start at the beginning of the list and find the MSE of the line formed by successive pixels until the MSE is greater than a predetermined threshold. Then start the next line segment at the pixel where the previous line ended. The MSE threshold, determined by experimentation, used for extracting the lines from the aerial images was 0.8 pixels squared. This value was fixed for all the images used for training and testing, as was the case for the intensity threshold.

By comparing the extracted lines and the original images shown in Figs. 4 to 6 it can be seen that proposed line extraction algorithm operates correctly. Table 3 shows that the average time to extract all the lines from a $512 \times 512$ grayscale image using $600-\mathrm{MHz}$. Pentium III processor is 0.0917 seconds. This is less than $5 \%$ of the total classification time.

\subsection{Line-feature Distribution Algorithm}

In order to classify an individual pixel, its relationship to the extracted lines must be known. There are two ways of correlating the line information with the individual pixels. The first way is to extract all the lines, plot them and then use a large window to find which lines are near the pixel. One advantage of this approach is that it uses memory efficiently and allows for the generation of statistical parameters for the lines within the window. The problem is that this approach would require a large window of approximately $50 \times$ 50 pixels and thus would result in a high computation cost. Clever coding could reduce this computation cost somewhat but even still the cost will be greater than the second method described below.

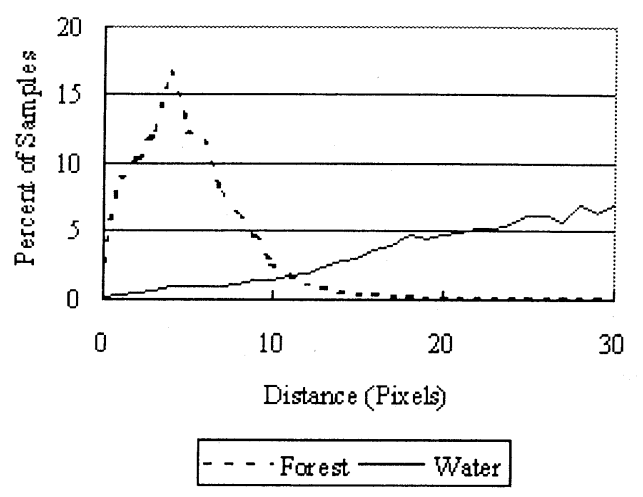

Fig. 1 Distance feature histogram.

The method used in this paper to correlate the individual pixels with the extracted lines is to distribute the line's features to the neighboring pixels as the lines are being extracted. This approach increases the speed of the classification at the cost of memory, as the linefeatures of several lines must be stored for each pixel in the image. In the proposed implementation of this approach an array was created to store the slope, the length, and the distance from the line to the pixel, for the five closest lines to the pixel. Two other arrays were created to store the number of lines influencing the pixel, and the distance from the pixel to the farthest line. Thus seventeen additional arrays of the same size of the original image are needed.

When a line is extracted, its features are distributed based on the length of the line. If the line is less than fifteen pixels in length then its features will be distributed perpendicularly to the line's length and radially from the two ends over a distance equal to twice the length of the line. If the length is greater than fifteen pixels then its features will be distributed in a similar manner over a distance of thirty pixels. If five lines are already influencing the pixel then two possible situations can occur. If the distance to the present line is greater that the distance to the other five lines, then the new line's features will be neglected. However, if it is closer, then it will replace the farthest line from the pixel.

Intuitively, a feature set consisting of intensity and line features would seem to be reasonable for separating the five classes. However, one element of the feature set on its own cannot perform this separation and therefore various elements of the feature set must be used. For example intensity can be used to separate water from fields, but a different feature is needed to separate water from forests. From the histogram shown in Fig.1 
Table 2 Classes resolved by each feature.

\begin{tabular}{c|c|c}
\hline \hline Feature & Resolved Class 1 & Resolved Class 2 \\
\hline number of lines & water & buildings/roads \\
\hline intensity & shadow & fields/beaches \\
\hline maximum lenght & fields/beaches & buildings/roads \\
\hline maximum distance & shadow & water \\
\hline slope & buildings/roads & forests \\
\hline distance & forests & water \\
\hline length & fields/beaches & forests
\end{tabular}

Table 3 Experimental results

\begin{tabular}{c|c|c|c|c}
\hline & Fig.4 & Fig.5 & Fig. 6 & Ave. \\
\hline Line Extraction (s) & 0.0584 & 0.1166 & 0.1002 & 0.0917 \\
\hline Line-feature Distribution (s) & 0.9906 & 2.3724 & 1.8188 & 1.7273 \\
\hline Classification (s) & 0.25 & 0.424 & 0.398 & 0.3573 \\
\hline Total (s) & 1.299 & 2.913 & 2.317 & 2.1763 \\
\hline Lines & 2249 & 6420 & 4201 & 4290 \\
\hline Error (\%) & 16.89 & 13.79 & 22.11 & 17.6 \\
\hline
\end{tabular}

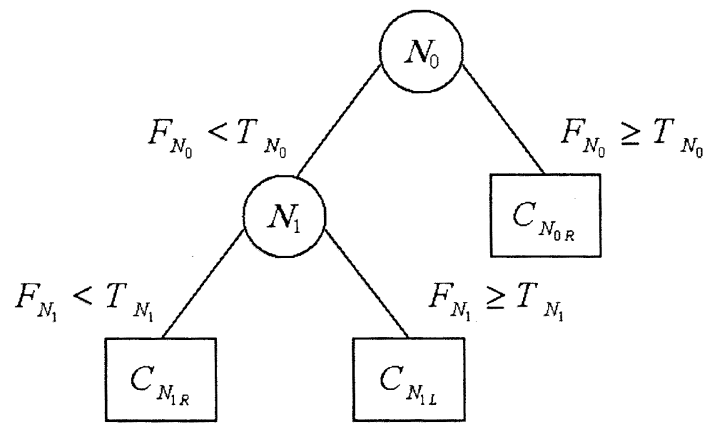

Fig. 2 Classification tree example.

it can be seen that the distance from the pixel to the line shows a clear difference between the classes. It is possible to show other similar histograms that can be used to separate other classes. These histograms have been summarized in Table 2 .

Although this technique is faster than a direct windowing approach it still has a high computation cost as can be seen from Table 3 . The average time for correlating the line information with the individual pixels is 1.7273 seconds and accounts for almost $80 \%$ of the classification time.

\section{4 Classification Tree and Training}

Any of the traditional classifiers can be used to classify the image provided that the feature set is adequate. A classification tree with single variable splits was chosen because it has many properties that are desirable for the proposed algorithm. In order to explain these properties it is necessary to understand how a classification tree is constructed.

Fig. 2 shows the construction of a simple classification tree with single variable splits ${ }^{22)}$. In this figure the algorithm begins at node $N_{0}$, which is called the root node. Here it tests feature $F_{N_{0}}$, which is a feature of the feature set. If the value of $F_{N_{0}}$ is greater than or equal to the threshold $T_{N_{0}}$, the algorithm will go to the terminal node $C_{N_{0 R}}$ and the algorithm will classify the sample as the class of the terminal node. However if $F_{N_{0}}$ is less than the threshold, then the algorithm will proceed to node $N_{1}$, which is called a child node. Here a similar test will be performed with feature, $F_{N_{1}}$, and threshold, $T_{N_{1}}$, and based on the test the algorithm will classify the sample as either $C_{N_{1 R}}$ or $C_{N_{1 L}}$. It should be noted that feature $F_{N_{0}}$, and feature, $F_{N_{1}}$ may be any feature of the feature set and may even be the same feature, and that all features and thresholds were determined by the training. The classification tree shown in Fig. 2 is a simple example and for most applications there are many more branches in the tree.

From the explanation of classification trees given above it is clear that this method of classification is very fast. Since the object of this paper is to classify aerial images quickly it seems to be a good choice of classifier. Also theoretical studies have shown ${ }^{23)}$ that the tree classifier is an accurate classifier if it is properly trained.

The training of the classifier is critical for its performance and the classification tree used in this paper was trained using the conventional method ${ }^{22)}$. The training principle for classification trees is to choose the splits so that each branch in the tree results in a lower impurity. Impurity is defined as the sum of the error for both child nodes. To find the best splitting threshold using the method of single variable splits, the impurity of all possible splits is calculated for all the samples entering the node and the split that produces the lowest impurity is chosen as the split for the node. To find the best split for subsequent nodes, only the samples that enter the node are used to find the split. Thus all the samples must be examined for the root node, while at each successive node a smaller number of samples needs to be examined. This procedure is repeated until the impurity level is lower than the desired level. This training method has a very desirable property for the proposed application in that the training is robust to moderate levels of noise in the training samples ${ }^{23)}$. Since it is difficult to classify an aerial image by hand with complete accuracy, this advantage in the training is very important.

In choosing the feature set for the proposed application three factors were considered to insure that the tree would be independent of the training images. First, the features must be invariant to translation, which is guaranteed as the pixel coordinates are not variables of 


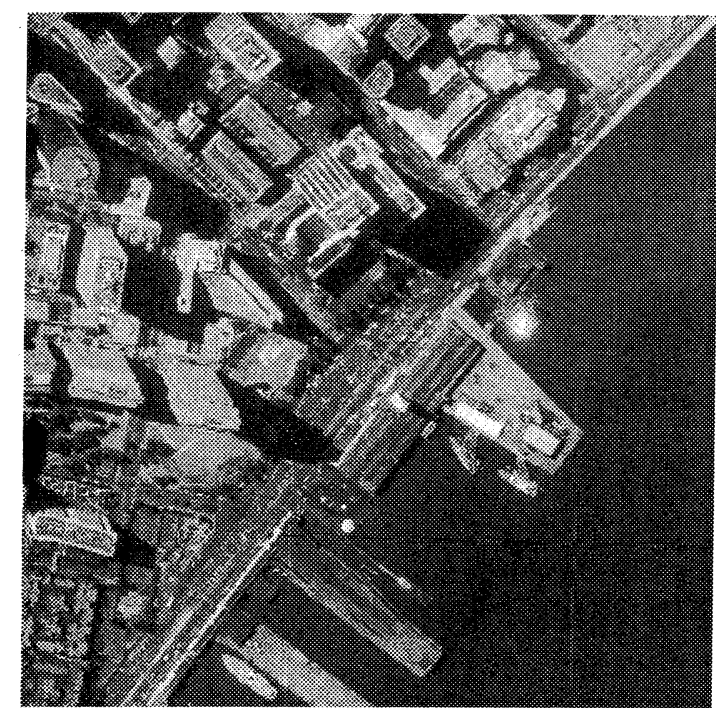

(a) urban and water $(768 \times 768)$

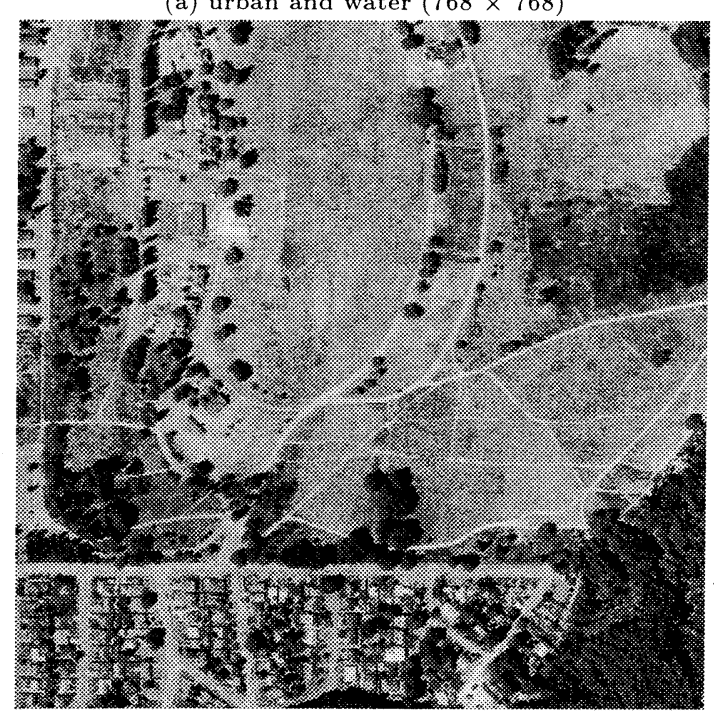

(c) suburb and field $(768 \times 768)$

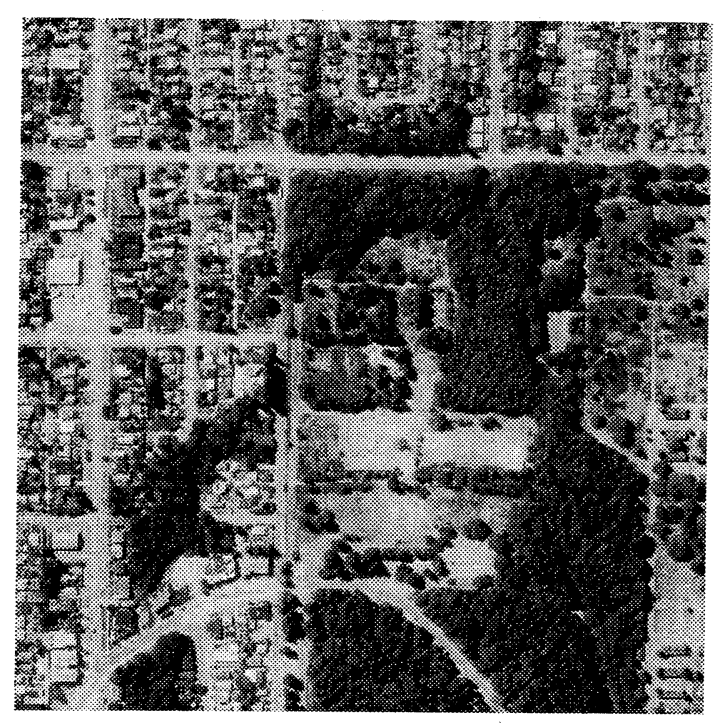

(b) suburb and forest $(768 \times 768)$

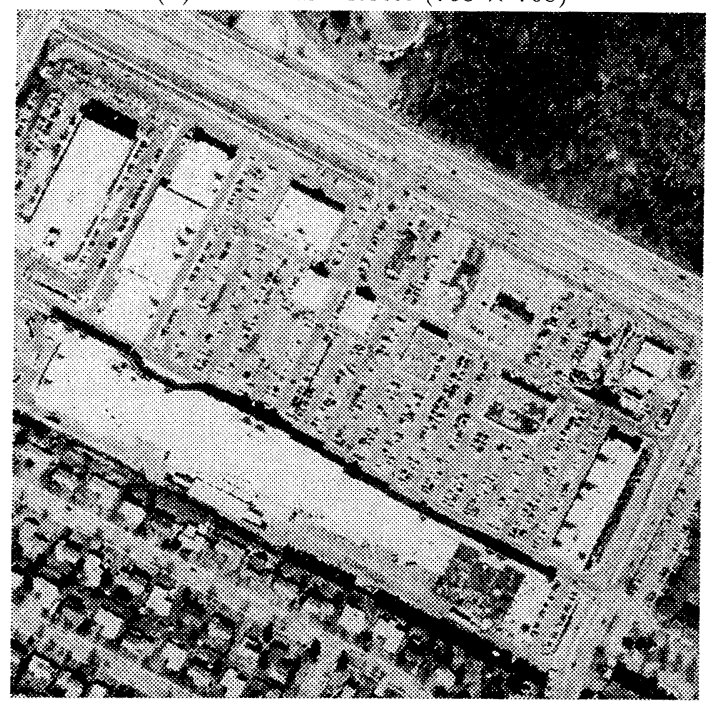

(d) suburb $(500 \times 500)$

Fig. 3 Training images.

the feature set. Second, the features must be invariant to scale, which is a property of classification trees with single variable splits ${ }^{22)}$. This means that if one feature is scaled a corresponding scale in the decision rule will compensate for the change. Since the resolution of most satellite images is known, this will not present a problem. Finally, the features must be invariant to rotation. To make the feature set rotationally invariant the slopes of the extracted lines are normalized to the slope of the longest line influencing the pixel.

To train the classifier, representative regions of the five classes were chosen from the four images shown in Fig.3. Three of these images are $768 \times 768$ grayscale images and one is a $500 \times 500$ grayscale image. The total number of samples in the training set was $1,642,936$ and the tree was trained until an error of $14 \%$ was obtained for the training samples. Further training could produce a tree with a much lower error for the training samples, however the resultant trees were over trained and produced poor results on the test images.

The resultant tree consisted of 143 nodes. As expected the intensity feature was the most dominant feature accounting for $17 \%$ of the decision nodes and $37 \%$ of the samples. Other dominant nodes were the number of lines, the length of the longest line, and the distance to the farthest line. From Table 3 it can be seen that the average classification time is 0.357 seconds or $16 \%$ of the total classification time.

\section{Experiments}

To verify the accuracy of the tree generated in Section 2.4 , three different images containing approximately equal quantities of each of the five classes were tested. The images are $512 \times 512$ grayscale images with 8 bits per pixel. These images are shown in Figs. 4 to 6 along 


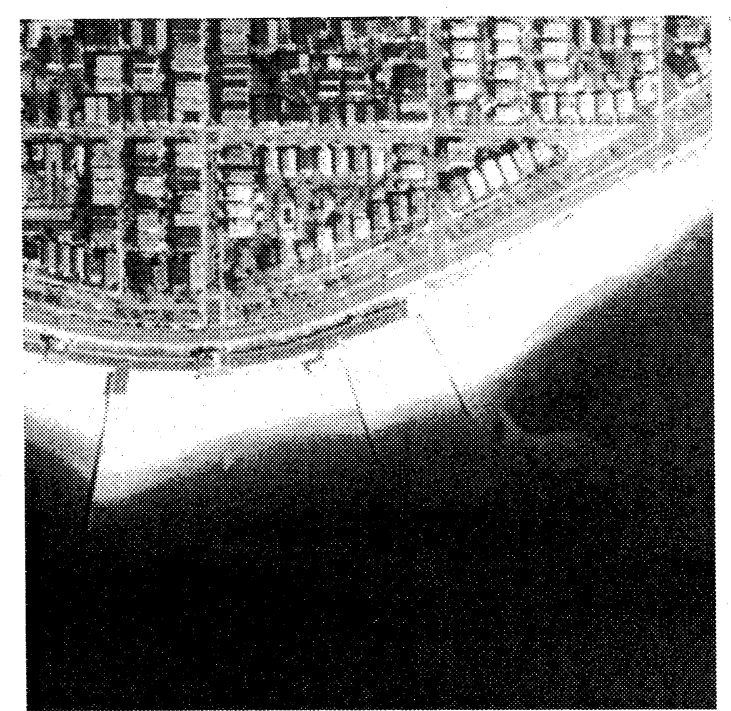

(a) coastal image

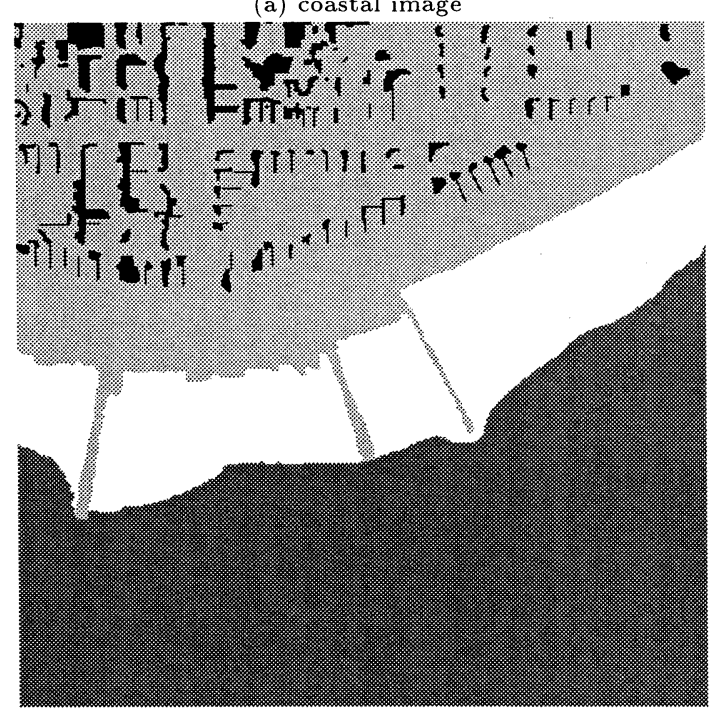

(c) hand-classified image

- Shadow $\mathbf{a}$ Forest

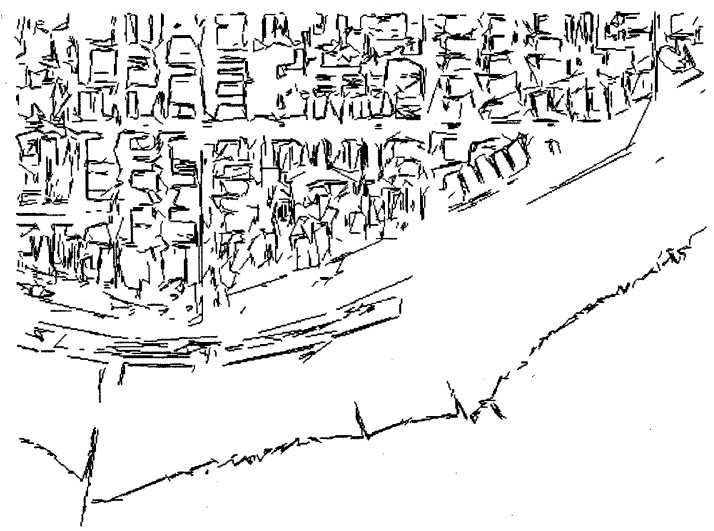

(b) extracted lines

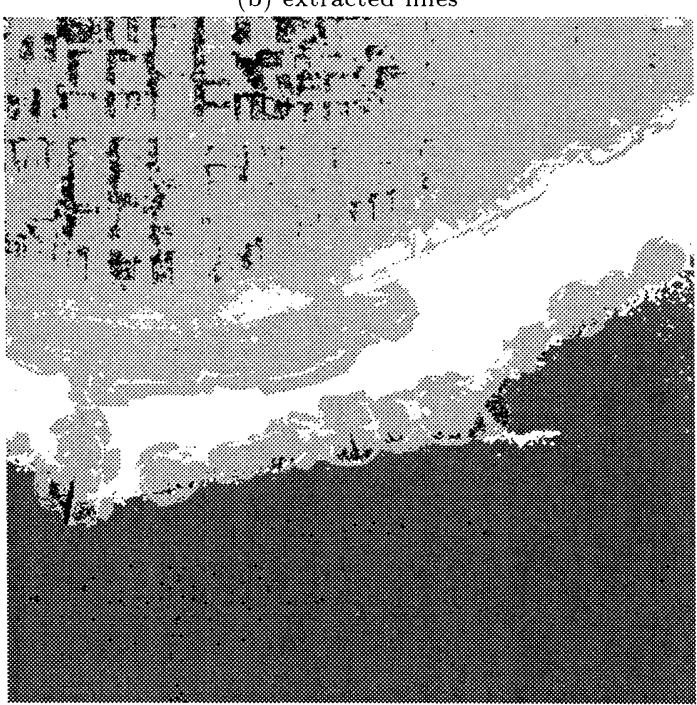

(d) classification results

Fig. 4 Coastal image

with the extracted line segments, the hand-classified image and the algorithm-classified image.

The first image, Fig.4, shows a coastal area. It was chosen to demonstrate the classes of water, field$\mathrm{s} /$ beaches and buildings/roads. The major source of error in this image occurred at the transitions between the beach and the ocean and the beach and the road, which suggests that the tree has not been adequately trained for classifying the fields/beaches class. From Table 3 it can be seen that the total classification time is 1.299 seconds with an average error of $16.89 \%$. This is the fastest classification time due to the large ocean area.

The second image, Fig.5, shows a purely urban scene and was chosen to demonstrate the classes of buildings/roads and shadow. The major source of error is the classification of some pixels in the roads as trees. As can be seen from Table 3 this image has an error of $13.79 \%$, which is the lowest error due to the relatively high contrast between the two classes present in the image. However, due to the high number of lines, this image also has the slowest classification time.

The third image, Fig.6, shows a rural scene and was chosen to demonstrate the classes of fields/beaches, forests, shadow, and buildings/roads. The major source of error in this image also occurred at the boundary between the field and other objects, as was the case in Fig.4. This image has an error of $22.11 \%$, which is the highest error and the image was also the most difficult to classify by hand.

From Table 3 it can be seen that the proposed classification algorithm has an average extraction time of 

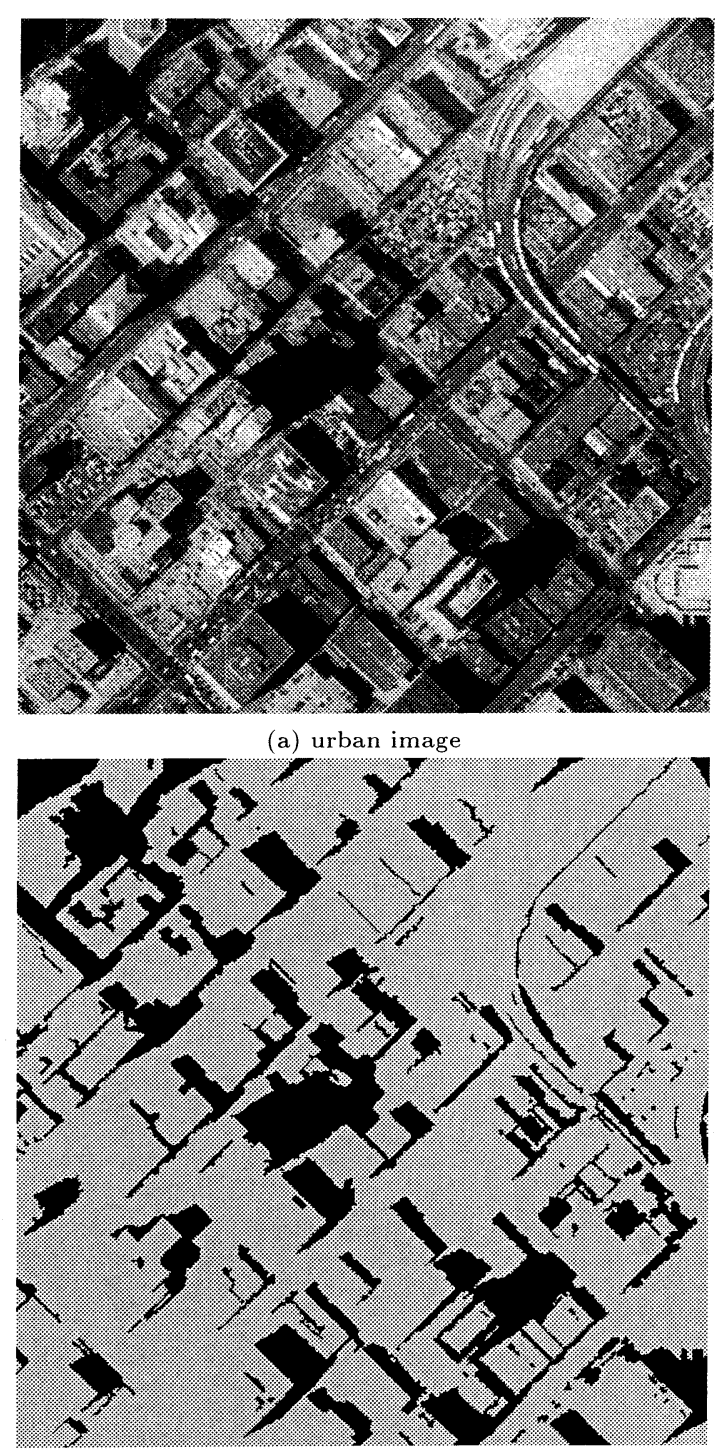

(c) hand-classified image

- Shadow Forest Water

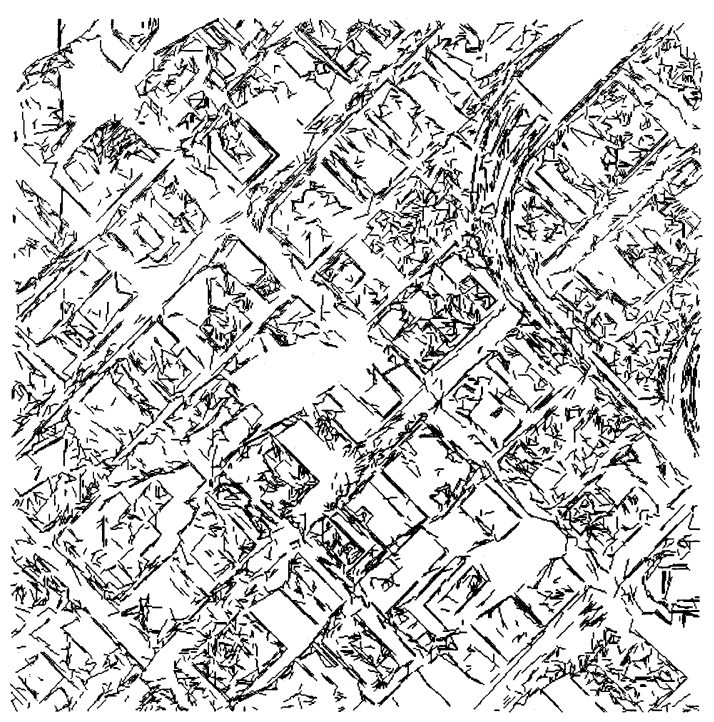

(b) extracted lines

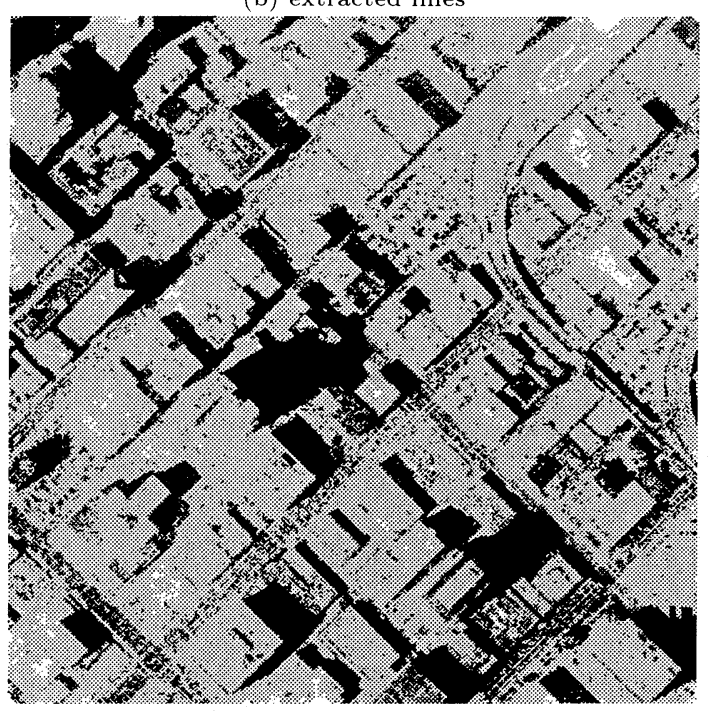

(d) classification results

Fig. 5 Urban image.

2.17 seconds and an average error of $17.6 \%$ for a fiveclass classification algorithm. There are not many aerial image classification algorithms presented in the literature. Most of these methods use neural networks ${ }^{14) 41 / 42)}$ or other techniques ${ }^{10) 43(44)}$. In order to compare the algorithm proposed in this paper with those available in the literature the two-class aerial image classification algorithm ${ }^{10)}$ was used. This algorithm was recently published and more closely resembles the proposed algorithm than the neural network approaches. This two-class aerial image classification algorithm had an average classification time of 59 seconds (19.7 seconds for the same processor) and an average error of $18.8 \%$. When compared with the results for the proposed algorithm it can be seen that the proposed algorithm performs better. Although their error seems close to the error of the proposed algorithm, the smaller number of classes makes it much higher than the proposed classification algorithm. Also the two-class algorithm only performed one classification for each block of 16 pixels, thus the actual classification speed is also much slower than the proposed algorithm.

\section{Conclusion}

In this paper we have presented a new approach to image classification. By using line-features extracted by a novel high-speed line extraction algorithm and a conventional classification tree, a fast, multi-class image classification algorithm has been developed. The classification algorithm was tested on $512 \times 512$ grayscale aerial images and an average classification time of 2.17 seconds was obtained using a 600-MHz. Pentium III processor. The accuracy of the classification was $17.6 \%$. 


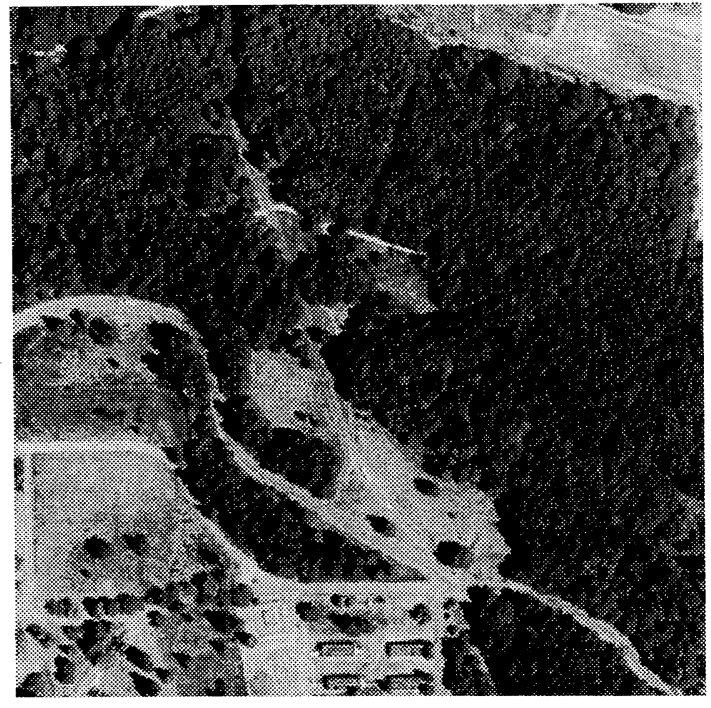

(a) rural image

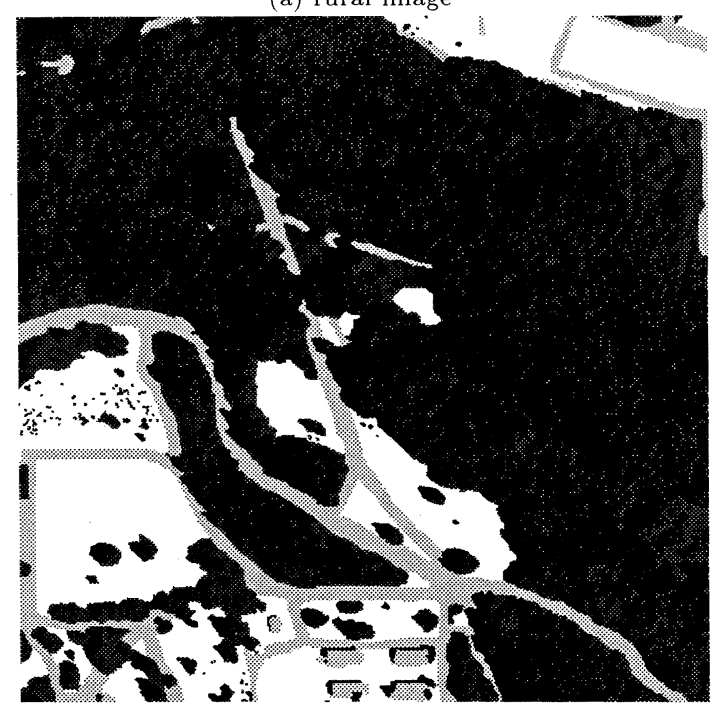

(c) hand-classified image

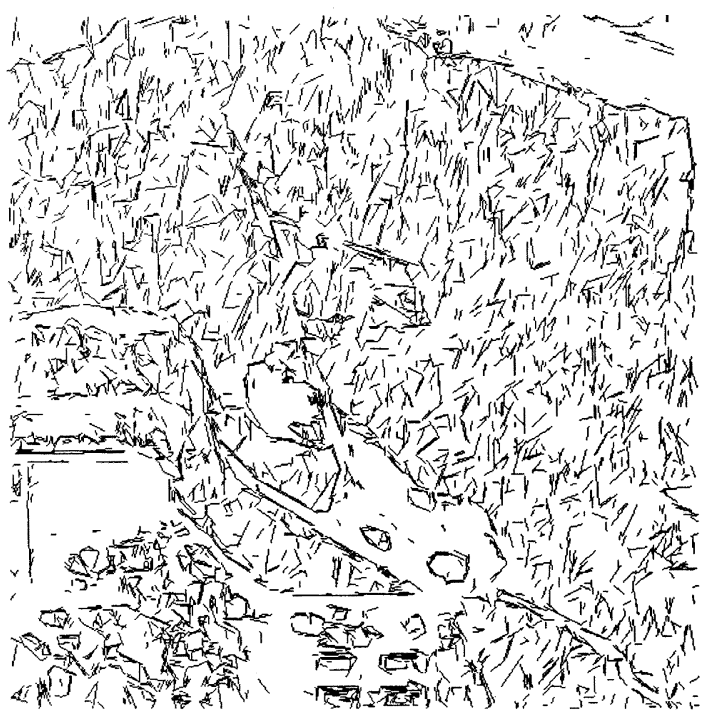

(b) extracted lines

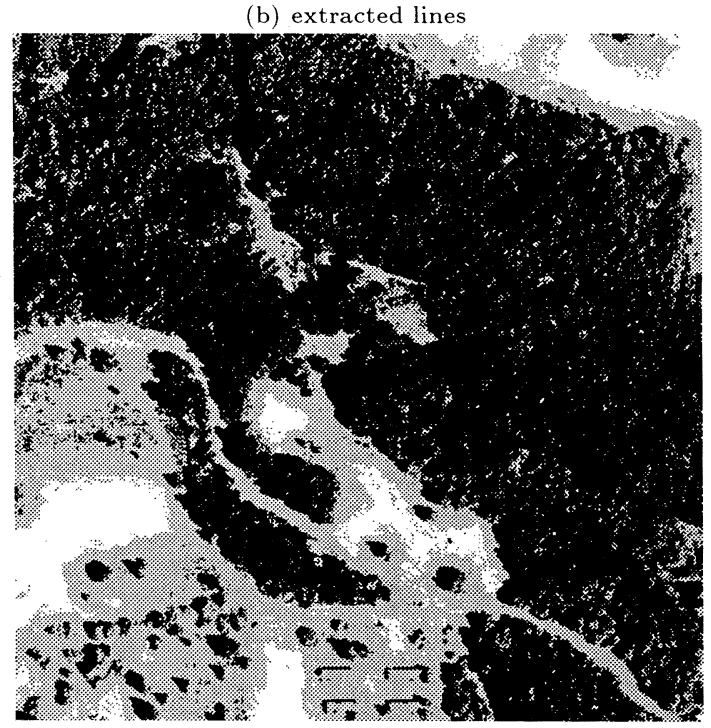

(d) classification results

- Shadow Forest Water 0 Building/Road $\square$ Field/Beach

Fig. 6 Rural image.

Due to the high-speed of the classification approach, it can be used to monitor changes in land use on a global scale, which requires processing vast amounts of aerial images. Also, by changing the training of the tree classifier, the classification approach can be easily adapted to many different applications.

\section{[References]}

1) H. Bischof, and A. Leonardis, "Finding optimal neural networks for land use classification," IEEE Trans. Geoscience and Remote Sensing, 36, 1, pp. 337-341 (Jan. 1998)

2) M. A. Hurn, K. V. Mardia, T. J. Hainsworth, J. Kirkbride, and E. Berry, "Bayesian fused classification of medical images," IEEE Trans. Medical Imaging, 15, 6, pp. 850-858 (Dec. 1996)

3) M. M. S. Chong, H. N. Tan, J. Liu, and R. K. L. Gay, "Geometric framework for fingerprint image classification," Pattern Recognition, 30, 9, pp. 1475-1488 (Sept. 1997)

4) N. Intrator, D. Reisfeld and Y. Yeshurun, "Face recognition using a hybrid supervised/unsupervised neural network," Pattern Recognition Letters, 17, 1, pp. 67-76 (Jan. 1996)

5) N. W. Campbell, W. P. J. Mackeown, B. T. Thomas, and T. Troscianko, "Interpreting Image Databases by Region Classifica- tion," Pattern Recognition, 30, 4, pp. 555-563 (Apr. 1997)

6) T. Y. Young, and T. W. Calvert, Classification, Estimation and Pattern Recognition. New York: American Elsevier Publishing Company Inc. (1974)

7) K. O. Perlmutter, S. M. Perlmutter, R. M. Gray, R. A. Olshen, and K. L. Oehler, "Bayes risk weighted vector quantization with posterior estimation for image compression and classification," IEEE Trans. Image Processing, 5, 2, pp. 347-360 (Feb. 1996)

8) C. S. Lindsey, and M. Stromberg, "Image classification using the frequencies of simple features," Pattern Recognition Letters, 21, 3, pp. 265-268 (Mar. 2000)

9) M. E. Hodgson, "What size window for image classification? A cognitive perspective," Photogrammetric Engineering and Remote Sensing, 64, 8, pp. 797-807 (1998)

10) J. Li, A. Najmi, and R. M. Gray, "Image classification by a twodimensional hidden Markov model," IEEE Trans. Signal Processing, 48, 2, pp. 517-533 (Feb. 2000)

11) A. Vailaya, A. Jain, and H. J. Zhang, "On image classification: city images vs. landscapes," Pattern Recognition, 31, 12, pp. 19211935 (Dec. 1998)

12) H. Q. Lu, and J. K. Aggarwal, "Applying perceptual organization to the detection of man-made objects in non-urban scenes," Pattern Recognition, 25, 8, pp. 835-853 (Aug. 1992)

13) Y. C. Tzeng, and K. S. Chen, "Fuzzy neural network to SAR image classification," IEEE Trans. Geoscience and Remote Sensing, 36, 1, pp. 301-307 (Jan. 1998) 
14) I. Kanellopoulos, and G. G. Wilkinson, "Strategies and best practice for neural network image classification," Int. J. of Remote Sensing, 18, 4, pp. 711-725 (Mar. 1997)

15) A. Jowik, S. Serpico, and F. Roli, "A parallel network of modified 1-NN and k-NN classifiers: Application to remote-sensing image classification," Pattern Recognition Letters, 19, 1, pp. 57-62 (May 1998)

16) J. Li, R. M. Gray, R. A. Olshen, "Multiresolution image classification by hierarchical modeling with two-dimensional hidden Markov models," IEEE Trans. Inf. Theory, 46, 5, pp. 1826-1841 (Aug. 2000)

17) Z. Kato, J. Zerubia, and M. Berthod, "Unsupervised parallel image classification using Markovian models," Pattern Recognition, 32, 4, pp. 591-604 (Apr. 1999)

18) M. Simard, S. S. Saatchi, and G. De Grandi, "Use of decision tree and multiscale texture for classification of JERS-1 SAR data over tropical forest," IEEE Trans. Geoscience and Remote Sensing, 38 5, pp. 2310-2321 (Sept. 2000)

19) G. Giacinto, and F. Roli, "An approach to the automatic design of multiple classifier systems," Pattern Recognition Letters, 22, 1, pp. 25-33 (Jan. 2001)

20) G. Giacinto, F. Roli, and G. Fumera, "Selection of image classifiers," Electron. Lett., 36, 5, pp. 420-422 (May 2000)

21) http://www.gsi.go.jp/ENGLISH/ABOUT/OUTLINE/ lt-plan.html

22) L. Breiman, J. H. Friedman, R. A. Olshen, and C. J. Stone, Classification and Regression Trees. Belmont, California: Wadsworth International Group (1984)

23) L. Devroye, L. Gyorfi, G. Lugosi, A Probabilistic Theory of Pattern Recognition. New York: Springer-Verlag New York Inc. (1996)

24) R. C. Gonzalez, and P. Wintz, Digital Image Processing. Second Edition. Reading Massachusetts: Addison-Wesley (1987)

25) D. H. Ballard, and C. M. Brown, Computer Vision. Englewood Cliffs, New Jersey: Prentice-Hall (1982)

26) D. H. Ballard, "Generalizing the Hough transform to detect arbitrary shapes," Pattern Recognition, 13, 2, pp. 111-122 (1981)

27) H. Li, M. A. Lavin, and R. J. LeMaster, "Fast Hough transform A hierarchical approach," Comput. Vision, Graphics, and Image Process., 36, pp. 139-161 (1986)

28) N. Guil, J. Villaba and E. L. Zapata, "A fast Hough transform for segment detection," IEEE Trans. Pattern Anal and Mach. Intell., 4, 11, pp. 1541-1548 (1995)

29) O. Chutatape, and L. Guo, "A modified Hough transform for line detection and its performance," Pattern Recognition, 32, 2, pp. 181-182 (1999)

30). Y. Zhang, and R. Webber, "A windowing approach to detecting line segments using Hough transform," Pattern Recognition, 29, 2, pp. 255-266 (1996)

31) J. Yuan, and C. Y. Suen, "An optimal $O(n)$ algorithm for identifying line segments from a sequence of chain codes," Pattern Recognition, 28, 5, pp. 635-646 (1995)

32) E. Bribiesca, "A new chain code," Pattern Recognition, 32, 2, pp 235-252 (1999)

33) K. Wall, and P. E. Danielsson, "A fast sequential method for polygonal approximation of digitized curves," Comput. Vision, Graphics, and Image Process., 36, pp. 139-161 (1986)

34) A. Pikaz, and I. Dinstein, "Optimal polygonal approximation of digital curves," Pattern Recognition, 28, 3, pp. 373-379 (1995)

35) J. W. Gates, M. Haseyama, and H. Kitajima, "A real-time line extraction method," IEEE Int. Symp. on Circuits and Systems'99, IV, pp. 68-71 (1999)

36) H. Kalviainen, P. Hirvonen, and E. Oja, "Houghtool - a software package for the use of the Hough transform," Pattern Recognition Letters, 17, pp. 889-897 (1996)

37) http://www.lut.fi./dep/tite/Xhoughtool/xhoughtool.html.

38) ftp.csd.uwo.ca/pub/papers/webber/Yuefeng/ HoughLineSegmentCode.c

39) H.R. Myler, and A.R. Weeks, The Pocket Handbook of Image Processing Algorithms in C. Englewood Cliffs, New Jersey: Prentice Hall (1993)

40) E. R. Dougherty, Probability and Statistics for the Engineering, Computing, and Physical Sciences. Englewood Cliffs, New Jersey: Prentice Hall (1990)

41) C. T. Lin, Y. C. Lee, and H. C. Pu, "Satellite sensor image classification using cascaded architecture of neural fuzzy network," IEEE Trans. Geoscience and Remote Sensing, 38, 2, pp. 10331043 (Feb. 2000)
42) J. Favela, and J. Torres, "A two-step approach to satellite image classification using fuzzy neural networks and the ID3 learning algorithm," Expert Systems with Applications, 14, 1, pp. 211-218 (Jan. 1998.)

43) C. Samson, L. Blanc-Feraud, G. Aubert, and J. Zerubia, "Variational model for image classification and restoration," IEEE Trans. Pattern Anal and Mach. Intell., 22, 5, pp. 460-472 (May 2000)

44) C. I. Chang, and C. Brumbley, "Kalman filtering approach to multispectral/hyperspectral image classification," IEEE Trans. Aerosp. and Electron. Syst., 35, 1, pp. 319-330 (Jan. 1999)

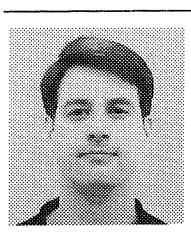

John Gates was born in Halifax, Canada in 1970. He received his Dip. Eng. from Dalhousie University, in Halifax in 1990 . He received his B.Eng and M.A.Sc. from the Technical University of Nova Scotia, Halifax, in 1993 and 1994 respectively. He is currently a doctoral candidate in the Information Engineering department of Hokkaido University, Japan. His research interests include integrated circuits and pattern recognition.

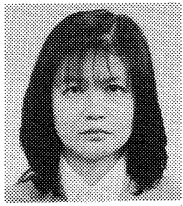

Miki Haseyama received the B.S. and M.S. degrees in electronics engineering from Hokkaido University, Japan, in 1986 and 1988, respectively. She was a research associate at Hokkaido University from August 1989 to January 1994 She is currently an Associate Professor at the same university. Her research interests are in signal processing and image processing.

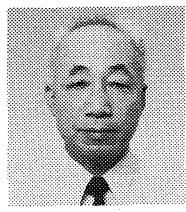

Hideo Kitajima was born in Osaka, Japan in 1942. He received the B.S. and M.S. degrees from Hokkaido University, in 1967 and 1969, respectively. In 1969 he became a research associate at Hokkaido University. He was advanced to the position of Associate Professor in 1982 . He is currently a professor at the same institution. His research interest includes signal processing, image processing, and image coding. 